\title{
RASGOS PROSÓDICOS DE LA EMOCIÓN: ESTUDIO DE UN CORPUS CONVERSACIONAL
}

\author{
Antonio Hidalgo Navarro \\ Departamento de Filología Española, Universitat de València, Grupo Val.Es.Co. \\ antonio.hidalgo@uv.es
}

Recibido: 15 de abril de 2020

Aceptado: 29 de octubre de 2020

\begin{abstract}
Resumen
El presente estudio describe algunas peculiaridades melódicas del habla emocional a través de un corpus conversacional donde se expresan diferentes valores emotivos. Como punto de referencia, partimos de la estrella de Plutchik (1980), con ocho emociones básicas (alegría, confianza, miedo, sorpresa, tristeza, disgusto, enojo y anticipación-interés) y dado el carácter limitado de nuestro corpus hemos reducido a cinco las emociones estudiadas (alegría, tristeza, enfado, miedo y sorpresa). Las marcas prosódicas seleccionadas, de acuerdo con investigaciones previas de otros autores, son el contorno del patrón melódico, asociado al tonema o a la inflexión final; el registro del contorno, esto es, su promedio de F0; la intensidad global del enunciado, como promedio de esta a lo largo del enunciado; y la velocidad de habla a través del enunciado (acto). A partir de aquí se ha realizado un análisis acústico y se proponen algunas conclusiones provisionales.
\end{abstract}

Palabras clave: Emoción, prosodia, funciones entonativas, conversación coloquial

\section{Resum}

El present estudi descriu algunes peculiaritats melòdiques de la parla emocional a partir d'un corpus conversacional en el qual s'expressen diferents valors emotius. Com a punt de referència, partim de l'estrella de Plutchik (1980), amb vuit emocions bàsiques (alegria, confiança, por, sorpresa, tristesa, disgust, enuig i anticipació-interès), de les quals, donat el caràcter limitat del nostre corpus, només n'estudiarem cinc (alegria, tristesa, enuig, por i sorpresa), que són les que hi han aparegut. Les marques prosòdiques seleccionades, d'acord amb investigacions prèvies, són el contorn del patró melòdic, associat al tonema o a la inflexió final; el registre del contorn, és a dir, la seva mitjana de F0; la intensitat global de l'enunciat (mitjana d'intensitat al llarg de l'enunciat); i la velocitat de parla en l'enunciat (acte). A partir d'aquí s'ha fet una anàlisi acústica i es proposen algunes conclusions provisionals.

Paraules clau: Emoció, prosòdia, funcions entonatives, conversa col·loquial

\begin{abstract}
This research describes some melodic peculiarities of emotional speech based on a conversational corpus in which different emotional values are expressed. As a landmark, we start with 8 basic emotions (joy, confidence, fear, surprise, sadness, displeasure, anger and anticipation-interest) from Plutchik's star (1980), which we have reduced to 5 (joy, sadness, anger, fear and surprise) according to those that have appeared caused by the limitations of the corpus. According to previous research, the selected prosodic features are the contour of the melodic patterns with its final inflection; the register of the contour, that is, its F0 average; the overall intensity of the statement, that is, the average of this throughout the statement; and the speed of speech through the statement (act). From that point on, an acoustic analysis has been carried out and some provisional conclusions are offered.
\end{abstract}

Keywords: Emotion, prosody, intonation functions, colloquial conversation 


\section{UBICACIÓN FUNCIONAL DE LA ENTONACIÓN "EMOCIONAL"}

Nuestro trabajo arranca con la consideración de ciertas preguntas de investigación:

- ¿A qué corresponde lo "emocional” en la comunicación humana?

- ¿Cómo estudiar la prosodia “emocional”? ¿Hay parámetros cuantificables (objetivables)?

- ¿A qué función prosódica se asocian los valores emotivos de la entonación?

Vayamos por partes. La primera cuestión podría reelaborarse con otra pregunta: ¿cuántas emociones cabe diferenciar entre las que se transmiten a través de la prosodia? Es decir, la ironía, el humor, la (des)cortesía... ¿están en el mismo nivel de "emocionalidad" que la alegría, el asco, la tristeza...? Sin pretender responder de forma definitiva a este problema de base, partimos de la conocida como "estrella de Plutchik" (Plutchik 1980) en la Figura 1, que ofrece una taxonomía de 8 emociones básicas a las que se contraponen otras 8 derivadas. De este modo, las emociones se pueden agrupar en ocho categorías primarias con funciones específicas en la vida humana: alegría ${ }^{1}$, confianza $^{2}$, miedo $^{3}$, sorpresa ${ }^{4}$, tristeza ${ }^{5}$, disgusto ${ }^{6}$, enojo ${ }^{7}$ y anticipación-interés ${ }^{8}$. Las demás emociones son, para Plutchik, combinaciones de las ocho anteriores que, al interactuar, permiten desarrollar el mapa de experiencias de cada individuo; de tales combinaciones se deriva una diversidad de emociones contrastadas por colores:

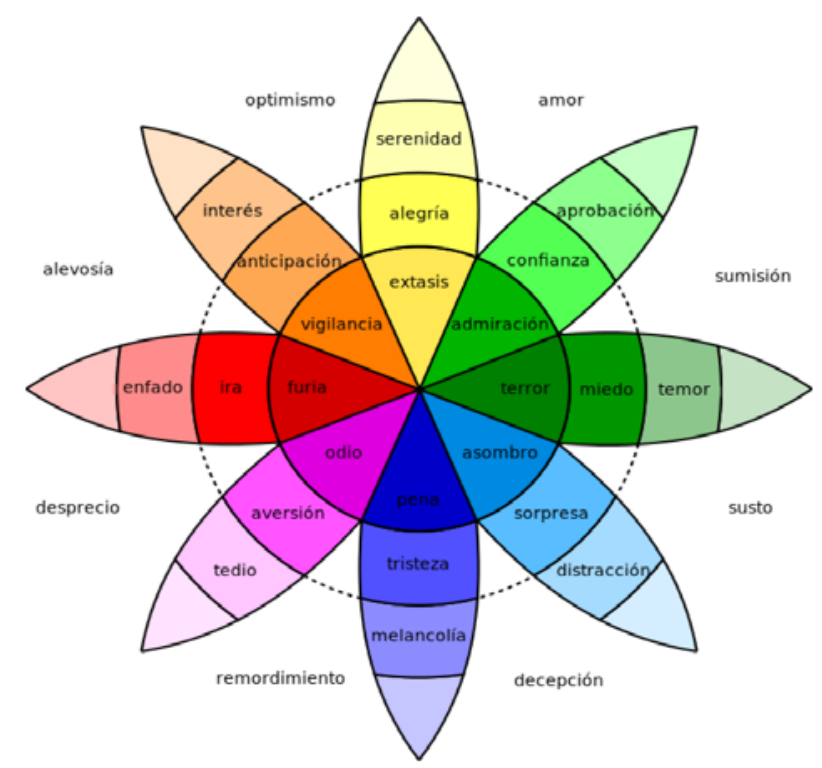

Figura 1. Rueda de emociones de Plutchik (1980).

\footnotetext{
${ }^{1}$ Emoción positiva, estado de bienestar y satisfacción respecto de uno mismo y/o de las condiciones de vida.

${ }^{2}$ Emoción que implica la creencia firme de que se puede actuar sin peligro de perjuicio o daño.

${ }^{3}$ En su grado más leve se manifiesta como aprensión (a modo de incertidumbre pesimista) y en un alto nivel puede convertirse en terror, estado que suele desplegar conductas de lucha o huida.

${ }^{4}$ Reacción ante circunstancias cambiantes e imprevisibles asociadas al entorno inmediato.

${ }^{5}$ Especie de "zozobra" que nos permite obtener el apoyo social de quienes la observan.

${ }^{6}$ Emoción sugerente de rechazo que de forma tenue puede expresarse como aburrimiento (ausencia de interés), y de forma más intensa como asco.

${ }^{7}$ Respuesta directa a una afrenta, especialmente cuando procede de la voluntad ofensiva de un tercero.

${ }^{8}$ Lo inverso de la sorpresa, es decir, la consideración de expectativas relevantes sobre lo venidero.
} 
A partir de aquí el problema radica en vincular el uso especializado de elementos prosódicos a las diversas emociones. En este sentido, podría decirse que ciertas marcas prosódicas apuntan a usos pragmáticos elaborados (ironía, atenuación, etc.) y obedecen a un uso más "consciente" o intencional por parte del emisor; frente a ello, los rasgos prosódicos emocionales, no serían controlados tan "conscientemente" por los hablantes y responderían a un grado de codificación más laxo.

Con la segunda pregunta tratamos de aclarar si la entonación "emocional" responde a algún "sistema" o si es mero producto de la variación de ánimo del hablante a cada momento y por tanto se reduce a lo idiosincrásico o asistemático. A esto podemos responder con alguna consideración: la expresión directa de emociones puede manifestarse mediante la entonación porque los músculos más sensibles y cercanos a los centros nerviosos experimentan de modo inmediato los efectos emotivos y los trasladan a la fonación (y por tanto, a la prosodia); no obstante, el estudio de estas variaciones prosódicas expresivo-emotivas resulta complejo porque las emociones y sentimientos posibles siguen sin estar claramente definidos (Fónagy y Magdics, 1963:313), como hemos apuntado previamente.

En cuanto a la tercera pregunta de investigación, ¿a qué función prosódica se asocian los valores emotivos de la entonación?, su respuesta exigiría previamente una descripción precisa de la emoción en el habla, trazando puentes entre lo propiamente emocional (tristeza, alegría, sorpresa, enfado, etc.) no codificado, y los significados fonopragmáticos (ironía, sarcasmo, (des)cortesía, humor, habla neutra, etc.) en apariencia semicodificados. En definitiva, se hace necesario explicar los ámbitos funcionales de la entonación en la conversación y sus niveles de actuación, para lo que proponemos la consideración del denominado modelo AIF (Análisis InteractivoFuncional) tal como se presenta en Hidalgo (2017) e Hidalgo (2019). Se trata, en definitiva, de esbozar (y si es posible establecer) diferentes grados de "sistematicidad" entre la diversidad funcional de la prosodia; puede plantearse así una distinción operativa entre:

- Prosodia "neutra" (+ objetiva)

- Prosodia espontánea ( \pm objetiva: determinada pragmática y contextualmente)

- Prosodia emocional (subjetiva: determinada ¿contextualmente? por el hablante)

Con estas premisas, en el siguiente punto trataremos de ubicar, funcionalmente hablando, la denominada entonación "emocional".

\section{HACIA UNA PROPUESTA DE UBICACIÓN: LA ENTONACIÓN "EMOCIONAL" DESDE EL MODELO AIF (ANÁLISIS INTERACTIVO- FUNCIONAL) ${ }^{9}$}

De acuerdo con el modelo AIF (Hidalgo, 2016, 2017, 2019), las funciones entonativas pueden organizarse en torno a dos ejes de relaciones, uno sintagmático y otro paradigmático que se proyectan respectivamente en los niveles monológico (intervenciones de un solo hablante) y dialógico (sucesión de intervenciones de más de un hablante) de la conversación.

\footnotetext{
9 El presente estudio ha sido realizado bajo el auspicio del Proyecto de Investigación EHSEEFONOEMOCIÓN / FFI2017-88310-P/MINECO, dirigido por X. Padilla García.
} 
En el nivel monológico cabe reconocer el siguiente haz de funciones entonativas:

- Funciones monológicas sintagmáticas. Sus ámbitos estructurales son el subacto y el acto $^{10}$ (combinación de subactos):

- Función demarcativa: establece la frontera lingüística entre actos

- Función integradora: determina la integración de subactos dentro de un acto. $\mathrm{Su}$ representación material es el grupo de entonación; otorga cohesión al discurso oral y proporciona información sobre la conclusión o continuidad del acto (segmenta, por ejemplo, las unidades internas de una serie enumerativa y permite reconocer sus diferentes componentes informativos)

- Funciones monológicas paradigmáticas. Su ámbito estructural es el acto (que posee curva melódica completa y una función comunicativa adecuada al contexto):

- Función modal primaria FMP. Los actos adquieren prosódicamente valores comunicativos "neutros" conmutables (aseveración, interrogación, volición) propios de registros cuidados (formales), como la lectura de textos escritos, discursos, conferencias, etc.

- Función modal secundaria FMS. La entonación actúa sobre unidades monológicas mínimas (actos) que se oponen por su valor pragmático.

Por lo demás, en el discurso conversacional espontáneo las intervenciones se suceden de modo que la comunicación se construye interactivamente a través de la colaboración mutua y sucesiva de hablante/-s y oyente/-s. La diversidad funcional de la prosodia debe abordarse así, necesariamente, desde una perspectiva más compleja, la dialógica, donde también se diferencian un eje funcional sintagmático y otro paradigmático que dan lugar a diferentes funciones:

- Funciones dialógicas sintagmáticas. En este ámbito se situaría la denominada entonación paralingüística de foco (Cantero, 2002; Cantero y Font-Rotchés, 2007; Cantero y Mateo, 2011; Cantero, 2014). Ocurre así, por ejemplo, en las topicalizaciones, cuando el hablante escinde un fragmento de habla con objeto de realzarlo informativamente, a la derecha o a la izquierda del cuerpo enunciativo nuclear. El empleo de una entonación específica contribuye a hacer más efectiva la emisión. Otra posibilidad en este ámbito funcional es la participación de la entonación en la regulación del sistema de alternancia de turnos: la entonación puede contribuir a organizar la sucesión de turnos conversacionales, en colaboración con otros recursos prosódicos y/o paralingüísticos como la cualidad de voz, los ajustes articulatorios, el tempo elocutivo, etc.

- Funciones dialógicas paradigmáticas. Reconocemos aquí la participación de la entonación paralingüística, asociada a ciertos rasgos entonativos no claramente codificados en el idioma (se sitúan más allá del código estrictamente lingüístico) y relacionados en muchos casos con la competencia sociocultural del hablante (Cantero, 2014:621). A la fijación de la modalidad enunciativa contribuyen entonces la entonación y elementos vocales muy diversos: diferentes modulaciones de la voz para expresar valores comunicativos cotidianos, aceleración/ralentización de la velocidad del habla, habla susurrada, etc. Tales rasgos no pueden ser tildados de idiosincrásicos, ya que los interlocutores los

${ }^{10}$ Unidades consideradas de acuerdo con la propuesta de segmentación de Briz y Val.Es.Co. (2003 y 2014). 
comparten formando un pseudocódigo o «código semiestable» (Cantero, 2014:621). En tales contextos habituales la prosodia contribuye a la construcción interactiva del sentido discursivo; hay varios casos significativos:

- Entonación y humor (Hidalgo, 2011:283-288), Existen situaciones discursivamente cerradas coincidentes con la presencia de risas, donde la prosodia contribuye a desencadenar efectos humorísticos ${ }^{11}$

- Entonación e ironía. La delimitación de las marcas prosódicas de ironía constituye un problema en el estado actual de la cuestión, ya que el contraste de patrones tonales en estos contextos va más allá de la frontera oracional, lo que exige una perspectiva compleja y multimodal del asunto (Attardo y otros, 2003; Padilla, 2004).

- Entonación y (des)cortesía. Esta interfaz resulta especialmente productiva en situaciones de contradicción entre hablantes (cuando alguien no quiere parecer tajante y recurre a parámetros bajos de F0, reduciendo la impresión de polémica), en contextos donde se expresan órdenes sin exigencia (parámetros mínimos de F0) o en construcciones sintácticas elípticas que "disfrazan" la intencionalidad del hablante.

La cuestión sería determinar cuál es la ubicación de la entonación emocional en este marco funcional. Puede entenderse como código semiestable relacionado con la competencia sociocultural de un hablante; permite transmitir afectividad, pero no pertenece al idioma (sistema), sino a la comunidad de habla o comunidad cultural. Sin embargo, estas consideraciones no despejan los conflictos de identificación funcional de la entonación emocional. Por ejemplo, en el ámbito de las funciones monológicas paradigmáticas, la exclamación subjetiva podría ser considerada como adscrita a la (FMS) o bien como forma de entonación emocional; en el ámbito de las funciones dialógicas sintagmáticas (FFDDSS) la focalización expresiva de algún segmento discursivo podría ser visto también como fenómeno de prosodia emotiva; en el ámbito de las funciones dialógicas paradigmáticas (FFDDPP) podríamos reconocer algunos usos prosódicos emotivos "comunes" (asociados a un código semiestable, de acuerdo con Cantero 2014) frente a casos de entonación emocional idiosincrásica, individual y, por tanto, asistemática (código "inestable")...

Proponemos así, en la Tabla 1, el mapa de funciones prosódicas en la conversación, donde quedan reflejadas las posibles interferencias ya mencionadas (y algunas otras):

\footnotetext{
${ }^{11}$ De acuerdo con Hidalgo (2011), puede tratarse tanto de magnitudes prosódicas elevadas (entonación exclamativa con rango tonal elevado; ampliación del campo tonal del hablante en el estilo directo; tono de falsete para imitar la voz femenina...) como de magnitudes prosódicas bajas (pronunciación parentética en susurro con intensidad mínima...).
} 


\begin{tabular}{|c|c|c|c|}
\hline PROSODIA “NEUTRA" & $\begin{array}{l}\text { PROSODIA } \\
\text { ESPONTÁNEA }\end{array}$ & $\begin{array}{l}\text { PROSODIA EMOCIONAL } \\
\text { "MANCOMUNADA" }\end{array}$ & $\begin{array}{l}\text { PROSODIA EMOCIONAL } \\
\text { IDIOSINCRÁSICA }\end{array}$ \\
\hline $\begin{array}{l}\text { - código estable } \\
\text { [sistemático/arbitrario] } \\
\text { - sin anclaje pragmático } \\
\text { - suelo prosódico }\end{array}$ & $\begin{array}{l}\text { - código semiestable } \\
\text { [+SISTEMÁTICO] } \\
\text { - con anclaje pragmático } \\
\text { - puede exigir recorrido } \\
\text { inferencial (interpretación) } \\
\text { adicional al oyente }\end{array}$ & $\begin{array}{l}\text { - código semiestable } \\
\text { [-SISTEMÁTICO] } \\
\text { - con anclaje pragmático } \\
\text { - no exige recorrido } \\
\text { inferencial (interpretación) } \\
\text { adicional al oyente }\end{array}$ & $\begin{array}{l}\text { - código inestable } \\
\text { [ASISTEMÁTICO] }\end{array}$ \\
\hline $\begin{array}{l}\text { FIVEVERACIÓN NEUTRA } \\
\text { FMP INTERROGACIÓN NEUTRA } \\
\text { VOLIONES PAÓN NEUTRA }\end{array}$ & $\begin{array}{l}\text { NIVEL MONOLÓGICO } \\
\text { FUNCIONES PARADIGMÁTICAS } \\
\begin{array}{l}\text { FMS } \\
\text { VARIANTES } \\
\text { DE LA FMP) }\end{array} \\
\text { NIVEL DIALÓGICO } \\
\text { INTERROGIÓN } \\
\text { EXCLAMACIÓN }\end{array}$ & 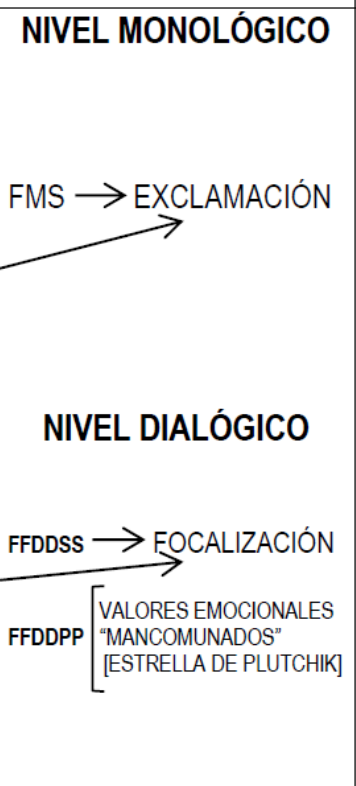 & \\
\hline
\end{tabular}

Tabla 1. Mapa de funciones entonativas en el Modelo AIF.

\section{ESTUDIO DE CORPUS}

\subsection{Aspectos metodológicos previos: corpus de referencia y unidad estructural de análisis (acto)}

El corpus de referencia del que partimos está constituido por tres conversaciones coloquiales del corpus Val.Es.Co. [C13, H25A1, H97A1]; la selección de sus informantes responde a los siguientes criterios de homogeneidad:

- 3 mujeres (una por conversación)

- nivel sociocultural medio-alto

- 25-45 años

- área metropolitana de Valencia

- monolingües (castellano)

- promedio de F0 (180/200 hz)

Las tres conversaciones cumplen los rasgos de la conversación coloquial (de acuerdo con Briz y Grupo Val.Es.Co., 1995, 2002):

- Oral: canal fónico.

- Dialogal: sucesión de intercambios.

- Inmediatez: se desarrolla en la coordenada espacio-temporal aquí-ahora-ante ti. 
- Retroalimentación y cooperación: la conversación se construye juntamente con otro/s y su/s intervención/es.

- Dinamismo: distribución no predeterminada de la alternancia de turnos.

- No planificación: escaso control de la producción de habla con presencia de reinicios, vacilaciones...

- Discurso no transaccional: fin interpersonal.

- Rasgos coloquiales: relación de igualdad social y/o funcional, relación vivencial de proximidad (conocimiento mutuo compartido), marco de interacción cotidiano y temática no especializada.

Por otra parte, los principios estructurales sobre los que reposa el análisis que hemos realizado parten del modelo de segmentación de Val.Es.Co. (Briz y Grupo Val.Es.Co., 2003, 2014), esto es, un sistema de unidades que tiene como unidad de referencia el acto (unidad mínima de acción e intención); así, es en el marco esta unidad donde hemos reconocido fragmentos de habla con entonación emocional. El acto se caracteriza por ser:

- aislable: posee fuerza ilocutiva propia, es decir, se trata de una unidad pragmática e intencional.

- identificable: hay marcas lingüísticas, prosódicas y semánticas, que marcan sus fronteras.

Además, un acto posee unidad melódica, es decir, un contorno melódico propio (o patrón entonativo, si se prefiere denominar así). De este modo, en su constitución interna el acto conforma un continuum de bloques informativos cohesionados prosódicamente. Así, el acto (1),

(1) Siempre tienes / laa la desviación profesional / la enfermedad profesional/ de observar / a los demás / y ahora / es- sentirse observado ess / una sensación extraña.

de acuerdo con su realización melódica, queda segmentado en ocho grupos de entonación (bloques informativos cohesionados prosódicamente), como vemos a continuación en (2):

(2) Siempre tienes $\rightarrow$ // laa la desviación profesional $\uparrow$ la enfermedad profesional $\uparrow /$ de observar $\uparrow$ a los demás $\uparrow / \mathrm{y}$ ahora $\uparrow$ es- sentirse observado ess / una sensación extraña. $\downarrow$

En su conjunto, (2) cumple los requisitos de la unidad acto: es aislable y representa una unidad comunicativa intencional (aseveración), y es identificable (presenta un patrón entonativo aseverativo, esto es, un contorno melódico descendente).

\subsection{Parámetros prosódicos estudiados (variables): curva melódica/tonema, registro (promedio de $\mathrm{F0}$ ), intensidad media, duración $/ \mathrm{n}^{0}$ de sílabas (velocidad de habla)}

Para configurar nuestra selección operativa de marcas prosódicas susceptibles de expresar emocionalidad, hemos contado con una bibliografía de base ${ }^{12}$. Así, de acuerdo

12 Destacan en este sentido, a nuestro entender, los trabajos de Adachi (1996), Álvarez y Blondet (2003), Anolli y otros (2000), Attardo y otros (2003), Cutler (1974), Hidalgo (2009), Hidalgo (2011), Hidalgo (2019), Kienpointer (1997), Léon (1970), Léon (1971), Léon (1972), Léon y Martín (1970), Martínez y 
con Léon (1971:47), existen diversos parámetros prosódicos que permiten caracterizar en alguna medida los rasgos emotivos de la prosodia:

- el contorno del patrón melódico permite reconocer el sentimiento expresado; en nuestro análisis tendremos en cuenta este factor a través de las variables curva melódica / tonema.

- el registro del patrón melódico puede tener un valor simbólico directo; así un registro + alto puede representar las emociones de alegría, timidez, ligereza..., un registro + bajo las emociones de tristeza, gravedad... En nuestro análisis consideramos este factor a través de la variable promedio de F0 del acto.

- la intensidad del patrón melódico evoca directamente la magnitud del sentimiento expresado; hemos considerado este factor en la variable promedio de intensidad del acto.

A las variables mencionadas hemos añadido la variable duración ( ${ }^{\circ}$ de sílabas por segundo de cada acto), entendida así como velocidad de habla.

\subsection{Selección y análisis melódico de casos: lo neutro, alegría, enfado, miedo, sorpresa, tristeza}

El procedimiento de análisis seguido parte del reconocimiento consensuado de actos "neutros" y "emocionales" en el corpus por parte de tres investigadores del grupo Fonoemoción/FFI2017-88310-P (incluido el que firma este estudio), lo que ha proporcionado el siguiente corpus de trabajo:

- Lo neutro: 30 actos

- Alegría: 26 actos

- Enfado: 39 actos

- Miedo: 7 actos

- Sorpresa: 16 actos

- Tristeza: 21 actos

Una vez delimitado el corpus hemos procedido a su análisis acústico mediante el uso de Praat (http://www.fon.hum.uva.nl/praat/) y se han anotado en una hoja Excel los valores obtenidos en cada acto para cada una de las variables. A modo de ejemplo véase en la Tabla 2 el análisis en Excel de (3), acto de enfado 1 de la conversación H25A1:

(3) oyE/ ¿te pués creer $\uparrow$ que ya van dos sábados $\rightarrow$ / que de los números que salen $\rightarrow$ ni uno no tengo NI UNO.

\begin{tabular}{|c|c|c|c|c|c|c|c|}
\hline ejemplo & $\begin{array}{c}\text { curva } \\
\text { melódica }\end{array}$ & tonema & $\begin{array}{c}\text { registro } \\
\text { (promedio } \\
\text { F0) }\end{array}$ & $\begin{array}{c}\text { intensidad } \\
\text { (promedio) }\end{array}$ & $\begin{array}{c}\text { duración } \\
\text { (segundos) }\end{array}$ & $\begin{array}{c}\text { no de } \\
\text { sílabas }\end{array}$ & $\begin{array}{c}\text { velocidad de } \\
\text { habla (sílabas } \\
\text { por segundo) }\end{array}$ \\
\hline $\begin{array}{c}\text { H25a1 } \\
\text { (ACTO 1) }\end{array}$ & circunfleja & ascendente & 223 & 72 & 5,94 & 30 & 5 \\
\hline
\end{tabular}

Tabla 2. Análisis prosódico del acto de enfado 1 de la conversación H25A1.

Rojas (2011), Muecke (1978), Myers (1977), Padilla (2004), Padilla (2009), Padilla (2011), Schaffer (1981), Truesdale y Pell (2018), entre otros. 


\subsection{Análisis comparado de casos}

Una vez tabulados los valores prosódicos de cada uno de los actos, hemos procedido al estudio comparado de cada variable, tomando como referencia la expresión de "lo neutro", frente a las diferentes emociones consideradas en nuestro corpus (alegría, tristeza, enfado, miedo y sorpresa). Pasamos a continuación a exponer los resultados de dicho estudio comparativo.

\subsubsection{Lo neutro vs. Alegría}

\subsubsection{Curva melódica y tonema}

Con respecto al contorno del patrón melódico (variable curva melódica y tonema), el resultado de la comparación (en términos porcentuales) entre actos neutros y alegres nos lleva a concluir que, en general, existe mayor homogeneidad en la estructura contorno/tonema para la expresión de "lo neutro" que para la expresión de "alegría":

- Lo neutro: el $56 \%$ de actos poseen un contorno melódico en declinación y un tonema descendente, frente a un $44 \%$ de actos que presentan diferentes estructuras, ninguna de ellas predominante; hay, pues, tendencia clara a curva en declinación y tonema descendente.

- Alegría: existe una distribución más diversa de la variable curva melódica y el tonema: el $27 \%$ de actos presenta una curva melódica circunfleja y un tonema circunflejo, el $23 \%$ de actos la estructura circunfleja y tonema descendente, el $15 \%$ de actos la estructura circunfleja y tonema suspendido y el $35 \%$ de actos presenta diferentes estructuras, ninguna de ellas predominante; puede decirse que existe cierta tendencia a la curva circunfleja (con variación del cuerpo melódico) con tonema cambiante.

La Figura 2 expresa de forma gráfica lo que acabamos de señalar:

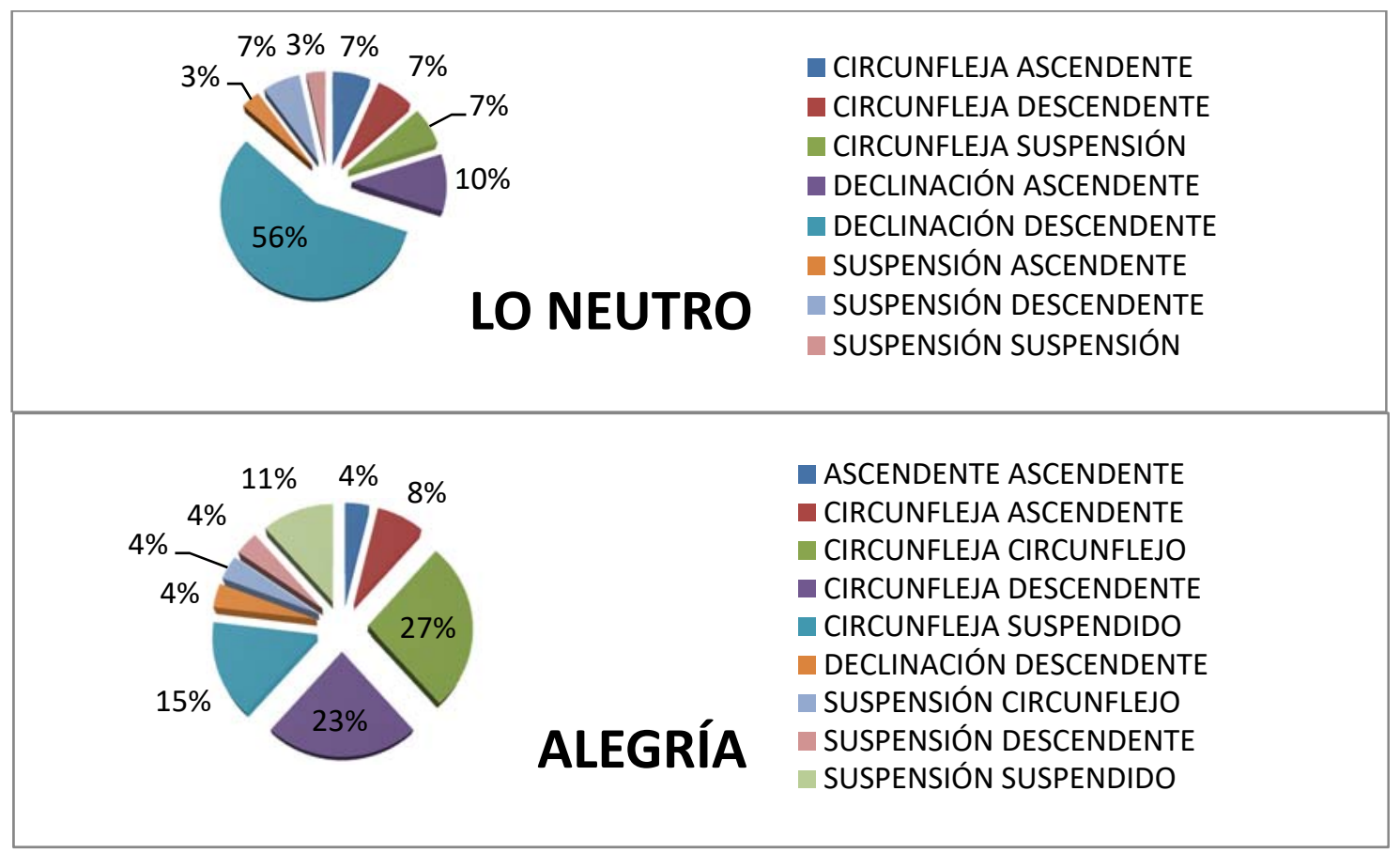

Figura 2. Distribución de curva melódica y tonema en actos neutros y de alegría. 


\subsubsection{Promedio de F0, promedio de Intensidad y velocidad de habla}

Con respecto al estudio comparativo de estos parámetros entre lo neutro y la expresión de alegría podemos afirmar que:

- el promedio de F0 es superior en alegría

- el promedio de Intensidad es superior en alegría

- la velocidad de habla resulta ligeramente inferior en alegría

En la Tabla 3 se representa gráficamente el resultado de la comparación entre actos neutros y alegres por lo que respecta a los promedios de F0 (en hercios) y de Intensidad (en decibelios) y a la velocidad de habla ( $\mathrm{n}^{\mathrm{o}}$ de sílabas por segundo):

\begin{tabular}{|c|c|}
\hline \multicolumn{2}{|c|}{ REGISTRO> PROMEDIO DE FO } \\
\hline LO NEUTRO & ALEGRÍA \\
\hline 221,5 & 240,5 \\
\hline \multicolumn{2}{|c|}{ INTENSIDAD MEDIA } \\
\hline LO NEUTRO & ALEGRÍA \\
\hline 83,7 & 85,1 \\
\hline DURACIÓN /No DE SÍLABAS (VELOCIDAD DE HABLA) \\
\hline LO NEUTRO & ALEGRÍA \\
\hline 6,1 & 5,5 \\
\hline
\end{tabular}

Tabla 3. Promedios de F0, de Intensidad y velocidad de habla en actos neutros y alegres.

\subsubsection{Lo neutro vs. Enfado}

\subsubsection{Curva melódica y tonema}

Con respecto al contorno del patrón melódico (variable curva melódica y tonema), el resultado de la comparación (en términos porcentuales) entre actos neutros y de enfado apunta a que, frente a "lo neutro", cuya tendencia a curva en declinación y tonema descendente ya ha sido indicada en 3.4.1.1., en los actos de enfado el $28 \%$ presenta una estructura circunfleja y tonema descendente, el 18\% una estructura circunfleja y tonema ascendente, el 13\% una estructura declinación y tonema descendente, el 13\% una estructura ascendente y tonema ascendente y el $21 \%$ de actos restantes ofrecen diferentes estructuras, ninguna de ellas predominante. La distribución de la curva melódica/tonema en enfado es, pues, de un $46 \%$ de actos con curva circunfleja y tonema variado (mayoritariamente descendente).

Este análisis se ve reflejado gráficamente en la Figura 3:

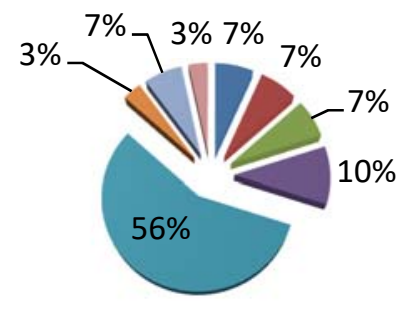
- CIRCUNFLEJA ASCENDENTE
- CIRCUNFLEJA DESCENDENTE
- CIRCUNFLEJA SUSPENSIÓN
- DECLINACIÓN ASCENDENTE
- DECLINACIÓN DESCENDENTE
SUSPENSIÓN ASCENDENTE
LO NEUTRO
- SUSPENSIÓN DESCENDENTE
- SUSPENSIÓN SUSPENSIÓN 


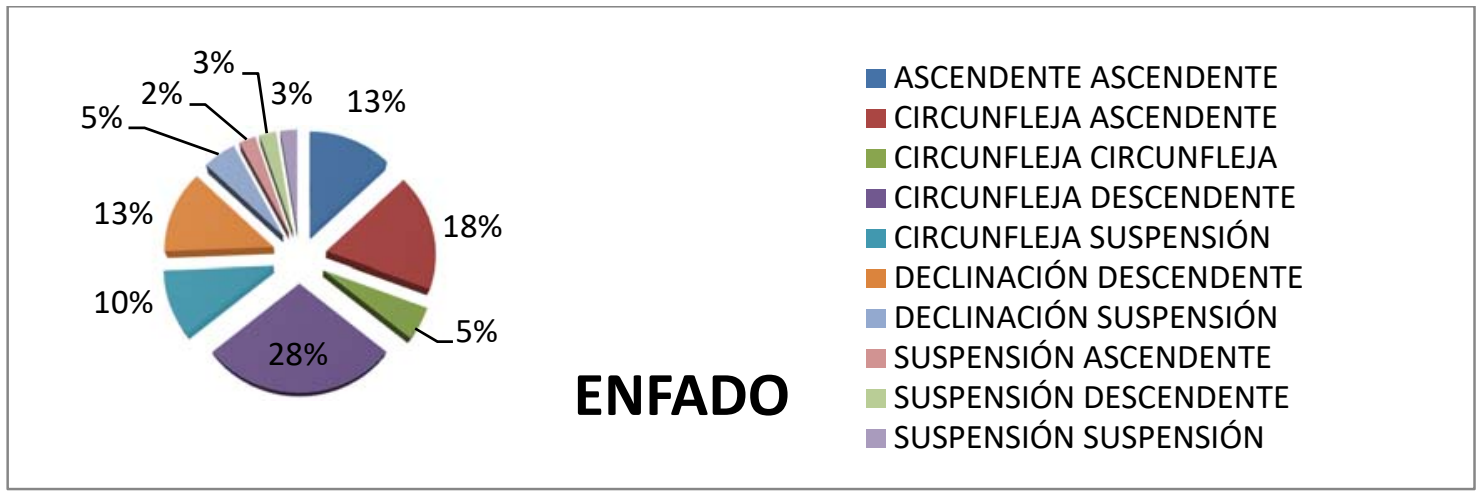

Figura 3. Distribución de curva melódica y tonema en actos neutros y de enfado.

\subsubsection{Promedio de F0, promedio de Intensidad y velocidad de habla}

Con respecto a estudio comparativo de estos parámetros entre lo neutro y la expresión de enfado podemos afirmar que:

- el promedio de F0 superior en enfado

- el promedio de Intensidad es inferior en enfado

- la velocidad de habla es similar en ambos casos

En la Tabla 4, se aporta el resultado de la comparación entre actos neutros y de enfado por lo que respecta a los promedios de F0 (en hercios) y de Intensidad (en decibelios), y a la velocidad de habla ( $\mathrm{n}^{\mathrm{o}}$ de sílabas por segundo):

\begin{tabular}{|c|c|}
\hline \multicolumn{2}{|c|}{ REGISTRO> PROMEDIO DE FO } \\
\hline LO NEUTRO & ENFADO \\
\hline 221,5 & 256,3 \\
\hline \multicolumn{2}{|c|}{ INTENSIDAD MEDIA } \\
\hline LO NEUTRO & ENFADO \\
\hline 83,7 & 77,4 \\
\hline DURACIÓN /No DE SÍLABAS (VELOCIDAD DE HABLA) \\
\hline LO NEUTRO & ENFADO \\
\hline 6,1 & 6,2 \\
\hline
\end{tabular}

Tabla 4. Promedios de F0, de Intensidad y velocidad de habla en actos neutros y de enfado.

\subsubsection{Lo neutro vs. Miedo}

\subsubsection{Curva melódica y tonema}

Aunque los ejemplos de miedo no son muchos respecto de las demás emociones estudiadas, como diferencia reseñable entre "lo neutro" y la expresión de miedo con respecto a este parámetro prosódico se observa que, frente a "lo neutro", con tendencia clara a curva en declinación y tonema descendente, la expresión de miedo ofrece un $29 \%$ de actos con estructura circunfleja y tonema ascendente y un $29 \%$ con estructura circunfleja y tonema descendente, lo que representa una tendencia del $58 \%$ de actos con curva circunfleja y tonema (equilibrado) ascendente o descendente.

El resultado de la comparación expresado en términos porcentuales entre actos neutros y de miedo queda reflejado en la Figura 4: 


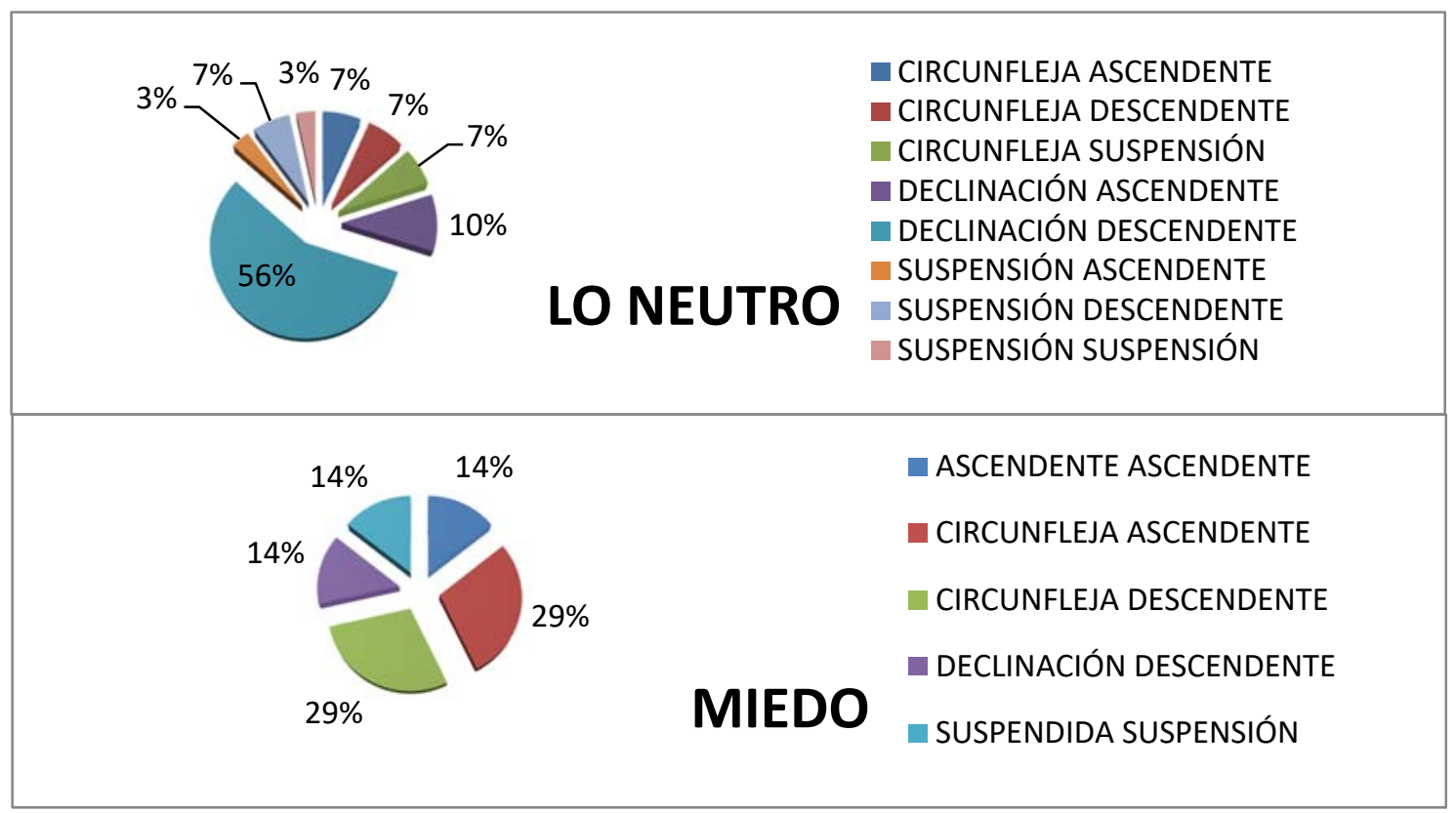

Figura 4. Distribución de curva melódica y tonema en actos neutros y de miedo.

\subsubsection{Promedio de F0, promedio de Intensidad y velocidad de habla}

En este ámbito la comparación entre lo neutro y la expresión de miedo indica que:

- el promedio de F0 es superior en miedo

- el promedio de Intensidad es inferior en miedo

- la velocidad de habla es inferior en miedo

En la Tabla 5, se puede ver el resultado de la comparación entre actos neutros y de miedo por lo que respecta a los promedios de F0 (en hercios) y de Intensidad (en decibelios), y a la velocidad de habla ( $\mathrm{n}^{\circ}$ de sílabas por segundo):

\begin{tabular}{|c|c|}
\hline \multicolumn{2}{|c|}{ REGISTRO> PROMEDIO DE FO } \\
\hline LO NEUTRO & MIEDO \\
\hline 221,5 & 269,7 \\
\hline \multicolumn{2}{|c|}{ INTENSIDAD MEDIA } \\
\hline LO NEUTRO & MIEDO \\
\hline 83,7 & 69,7 \\
\hline DURACIÓN /No DE SÍLABAS (VELOCIDAD DE HABLA) \\
\hline LO NEUTRO & MIEDO \\
\hline 6,1 & 5 \\
\hline
\end{tabular}

Tabla 5. Promedios de F0, de Intensidad y velocidad de habla en actos neutros y de miedo.

\subsubsection{Lo neutro vs. Sorpresa}

\subsubsection{Curva melódica y tonema}

Con respecto al contorno del patrón melódico (variable curva melódica y tonema), el resultado de la comparación expresado en términos porcentuales entre actos neutros y 
de sorpresa apunta a que frente a "lo neutro" (con tendencia clara a una curva en declinación y un tonema descendente), la expresión de sorpresa presenta un $31 \%$ de actos con estructura circunfleja y tonema descendente y un $25 \%$ de actos con estructura circunfleja y tonema ascendente, lo que ofrece como tendencia un $56 \%$ de actos de sorpresa con curva circunfleja y con tonema diferenciado (con ligero predominio del descendente).

El resultado puede visualizarse comparativamente en la Figura 5:

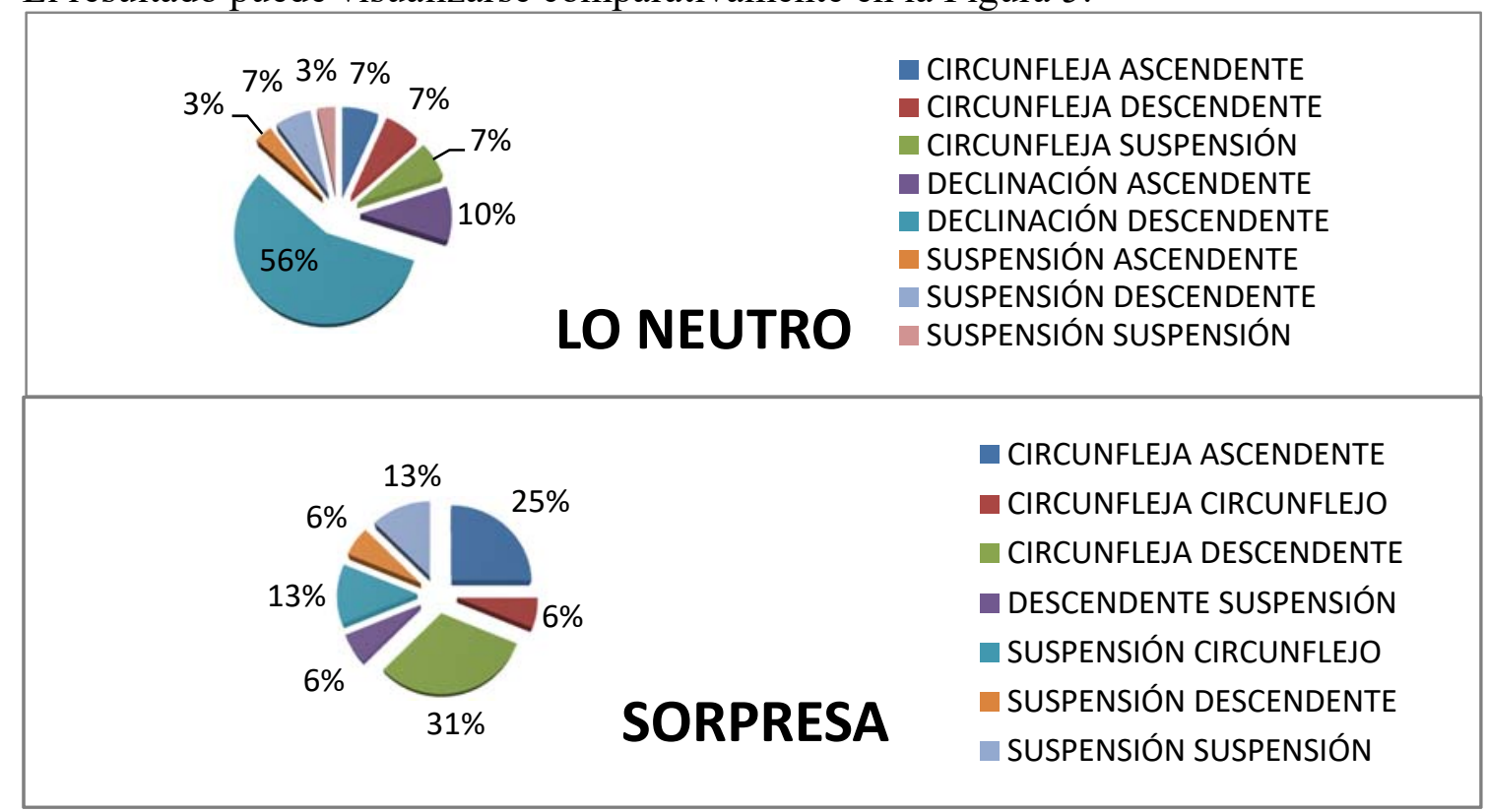

Figura 5. Distribución de curva melódica/tonema en actos neutros y de sorpresa.

\subsubsection{Promedio de F0, promedio de Intensidad y velocidad de habla}

La comparación entre lo neutro y la expresión de sorpresa indica que:

- el promedio de F0 es superior en sorpresa

- el promedio de intensidad es inferior en sorpresa

- la velocidad de habla es similar en lo neutro y en sorpresa

En la Tabla 6, se presenta el resultado de la comparación entre actos neutros y de sorpresa por lo que respecta a los promedios de F0 (en hercios) y de Intensidad (en decibelios), y a la velocidad de habla ( $\mathrm{n}^{\mathrm{o}}$ de sílabas por segundo):

\begin{tabular}{|c|c|}
\hline \multicolumn{2}{|c|}{ REGISTRO> PROMEDIO DE FO } \\
\hline LO NEUTRO & SORPRESA \\
\hline 221,5 & 246,6 \\
\hline \multicolumn{2}{|c|}{ INTENSIDAD MEDIA } \\
\hline LO NEUTRO & SORPRESA \\
\hline 83,7 & 77,6 \\
\hline DURACIÓN /No DE SÍLABAS (VELOCIDAD DE HABLA) \\
\hline LO NEUTRO & SORPRESA \\
\hline 6,1 & 5,6 \\
\hline
\end{tabular}

Tabla 6. Promedios de F0, de Intensidad y velocidad de habla en actos neutros y de sorpresa. 


\subsubsection{Lo neutro vs. Tristeza}

\subsubsection{Curva melódica y tonema}

Con respecto al contorno del patrón melódico (variable curva melódica y tonema), el resultado de la comparación expresado en términos porcentuales entre actos neutros y de tristeza indica que, frente a "lo neutro" (curva en declinación y tonema descendente), la expresión de tristeza se caracteriza por una estructura de curva melódica en suspensión y tonema suspendido en el $52 \%$ de actos, mientras que el $48 \%$ de actos restantes ofrecen diferentes estructuras, con predominio de curva en declinación y tonema descendente (en el $24 \%$ de casos).

El resultado puede visualizarse comparativamente en la Figura 6:

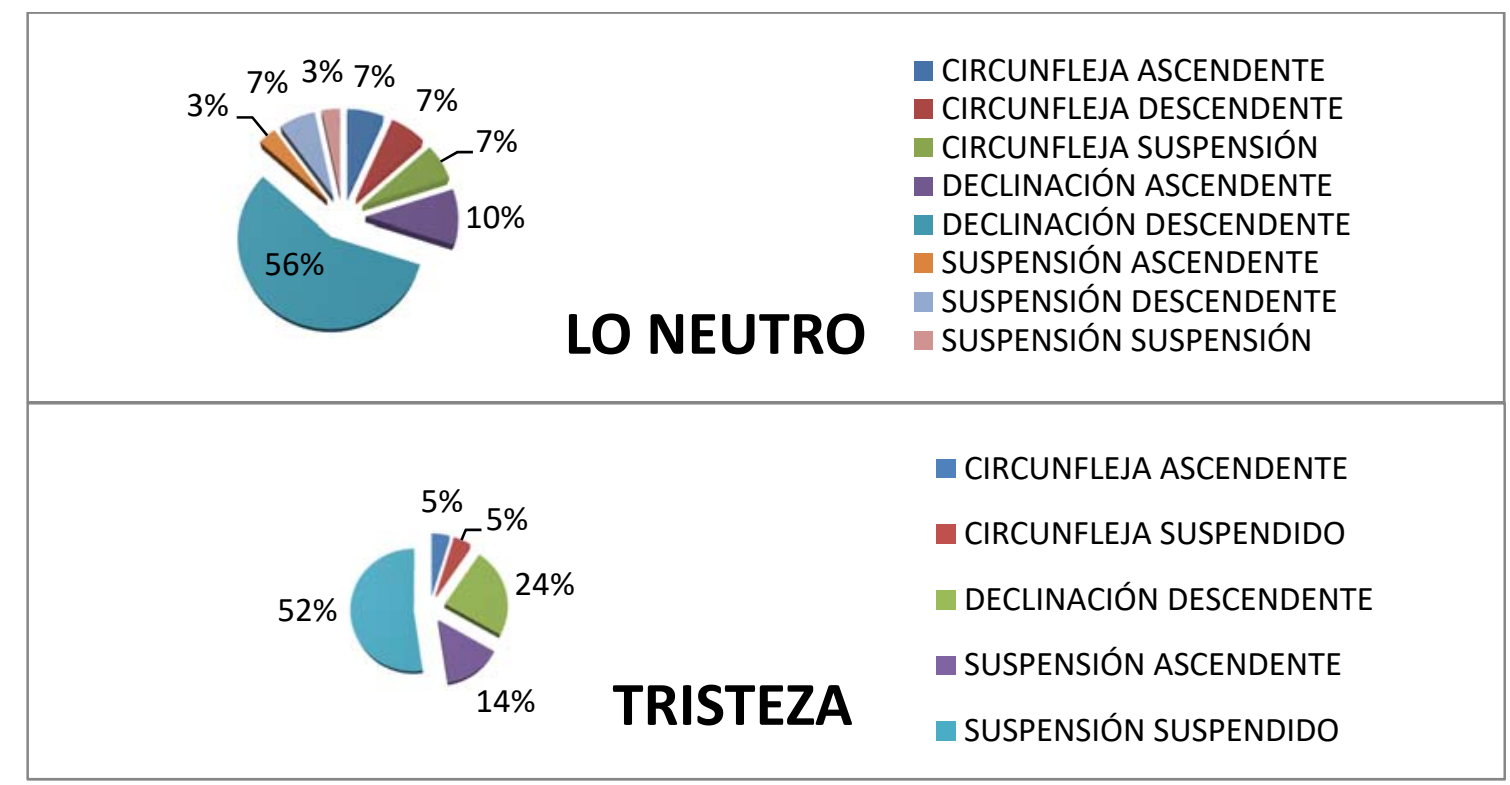

Figura 6. Distribución de curva melódica y tonema en actos neutros y de tristeza.

\subsubsection{Promedio de F0, promedio de Intensidad, velocidad de habla}

La comparación entre lo neutro y la expresión de tristeza indica con respecto a estos parámetros que:

- el promedio de F0 es inferior en tristeza

- el promedio de intensidad es inferior en tristeza

- la velocidad de habla es inferior en tristeza

En la Tabla 7, se aporta el resultado de la comparación entre actos neutros y de tristeza por lo que respecta a los promedios de F0 (en hercios) y de Intensidad (en decibelios), y a la velocidad de habla ( $\mathrm{n}^{\mathrm{o}}$ de sílabas por segundo): 


\begin{tabular}{|c|c|}
\hline \multicolumn{2}{|c|}{ REGISTRO> PROMEDIO DE FO } \\
\hline LO NEUTRO & TRISTEZA \\
\hline 221,5 & 179,1 \\
\hline \multicolumn{2}{|c|}{ INTENSIDAD MEDIA } \\
\hline LO NEUTRO & TRISTEZA \\
\hline 83,7 & 80,8 \\
\hline DURACIÓN /№ DE SÍLABAS (VELOCIDAD DE HABLA) \\
\hline LO NEUTRO & TRISTEZA \\
\hline 6,1 & 4 \\
\hline
\end{tabular}

Tabla 7. Promedios de F0, de Intensidad y velocidad de habla en actos neutros y de tristeza

\section{CONCLUSIONES: HACIA UN PROTOTIPO PROSÓDICO DE EMOCIONES}

Los resultados obtenidos a partir de un análisis acústico previo de parámetros prosódicos apuntan algunas tendencias que podrían permitirnos hablar de ciertos prototipos prosódicos para la expresión de las emociones consideradas en nuestro estudio (alegría, enfado, miedo, sorpresa y tristeza), comparando el comportamiento de esos mismos parámetros prosódicos con la expresión de lo neutro. La Figura 7 representa esquemáticamente las diferencias y afinidades entre dichos prototipos respecto de su intensidad media, registro (promedio de F0), curva melódica ${ }^{13} \mathrm{y}$ velocidad de habla.

La interpretación del esquema de la Figura 7 nos permite aportar ciertas conclusiones en nuestro estudio, advirtiendo no obstante de que se trata de un trabajo prospectivo, pendiente aún de validación cuantitativa y estadística:

- Las mayores divergencias prosódicas a la luz de nuestro corpus son las que se producen entre alegría y tristeza, que solo poseen como rasgo común el hecho de que su velocidad de habla es inferior a lo neutro; sus rasgos prosódicos restantes están claramente diferenciados.

- Por su parte, alegría y enfado (teniendo en cuenta que los rasgos prosódicos de enfado son similares a los de sorpresa) solo poseen como rasgo común el hecho de que su registro (promedio de F0) es superior al de "lo neutro". Los demás rasgos son claramente diferentes en cuanto a su tendencia.

- Existe afinidad prosódica entre alegría y miedo, de hecho, solo poseen como rasgo diferencial el de intensidad media, los demás rasgos son equivalentes en su tendencia

- Hay "identidad" prosódica entre enfado y sorpresa, ya que todos sus parámetros prosódicos coinciden.

\footnotetext{
${ }^{13}$ La etiqueta CIRCUNFLEJA X en la Figura 13 en relación con el tipo de curva melódica y tonema, indica que el carácter circunflejo del movimiento melódico puede venir dado por un ascenso-descenso o por un descenso-ascenso de la curva; lo relevante es, en cualquier caso, que exista curva circunfleja.
} 


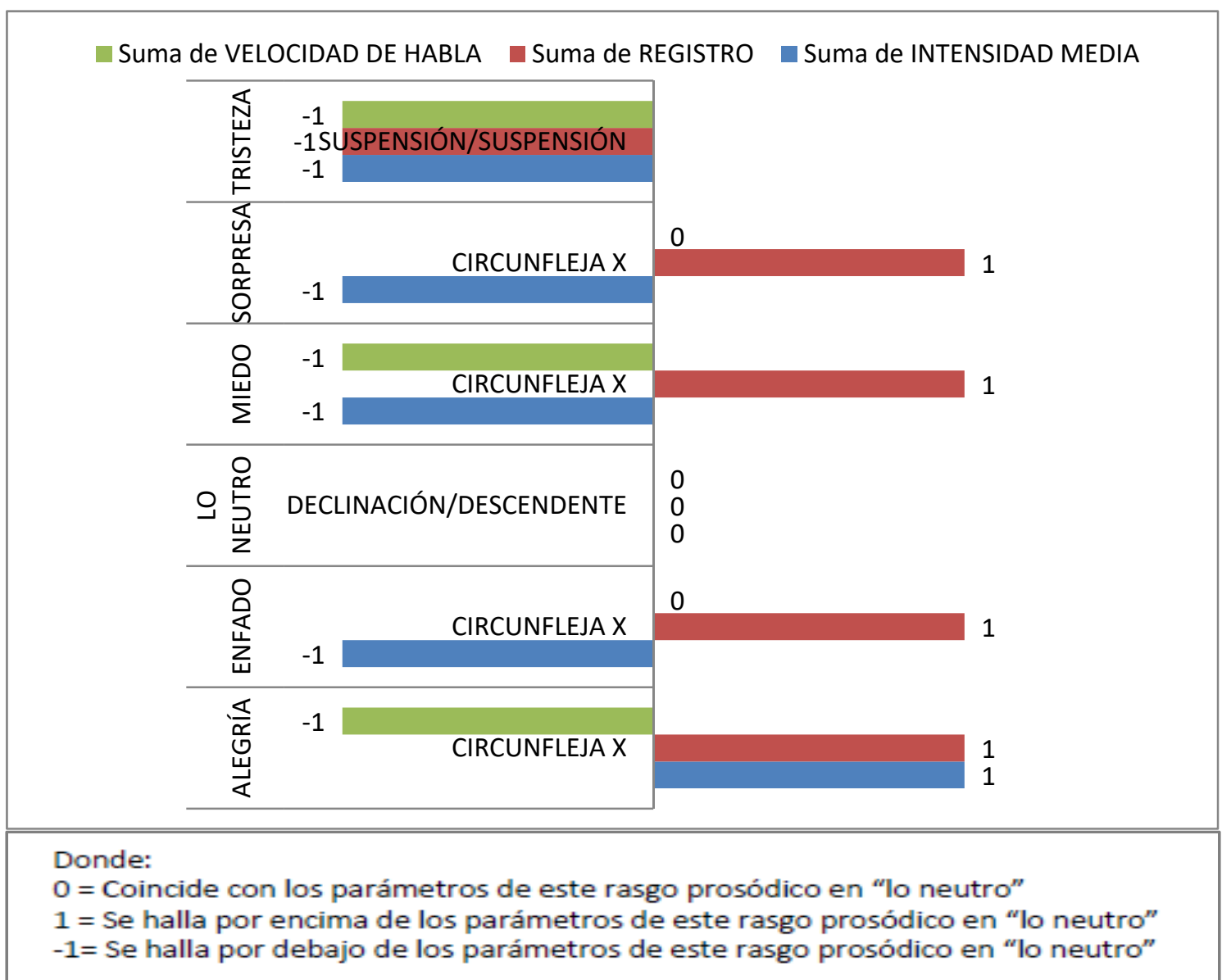

Figura 7. Representación esquemática de los prototipos prosódicos de alegría, enfado, miedo, sorpresa y tristeza, comparados con "lo neutro".

En conclusión, tomando como referencia "lo neutro":

- Las emociones de alegría vs. tristeza y alegría vs. enfado se expresan prosódicamente de formas muy diferentes, presentándose, pues, como emociones claramente opuestas.

- Alegría y miedo se valen de recursos prosódicos similares (curva/tonema, registro, velocidad de habla), al menos a la luz de nuestro corpus.

- Enfado y sorpresa también se expresan prosódicamente de forma similar.

\section{REFERENCIAS BIBLIOGRÁFICAS}

Adachi, Takanori (1996): «Sarcasm in Japanese». Studies in Language 20 (1), 1-36.

Álvarez, Alexandra; Blondet, María Alejandra. (2003): «Cortesía y prosodia: un estudio de la frase cortés en el español de Mérida (Venezuela)». En Martín Butragueño, Pedro; Herrera, Esther (eds.), La tonía. Dimensiones fonéticas y fonológicas. México: El Colegio de México, 319-330.

Anolli, Luigi; Ciceri, Rita; Infantino, Maria Giaele (2000): «Irony as a game of implicitness: Acoustic profiles of ironic communication». Journal of Psycholinguistic Research 29 (3), 275-311.

Attardo, Salvatore, Jodi Eisterhold, Jennifer Hay e Isabella Poggi (2003). «Multimodal markers of irony and sarcasm». Humor, 16(2), 143-260. 
Briz, Antonio y otros (2003): «Un sistema de unidades para el estudio del lenguaje coloquial». Oralia 6, 7-61.

Briz, Antonio y otros (2014): «Las unidades del discurso oral. La propuesta Val.Es.Co. de segmentación de la conversación (coloquial)». Estudios de Lingüística del Español 35, 13-73.

Cantero, Francisco José (2002): Teoría y análisis de la entonación. Barcelona: Edicions de la Universitat de Barcelona.

Cantero, Francisco José (2014): «Códigos de la entonación y entonación emocional». En Díaz, Ana; Fumero, $\mathrm{M}^{\mathrm{a}}$ del Carmen; Lojendio, $\mathrm{M}^{\mathrm{a}}$ Pilar; Burgess, Sally; Sosa; Eulalia; Cano, Antonio (eds.), Actas del XXXI Congreso Internacional AESLA. La Laguna: Universidad de La Laguna, 618-629.

Cantero, Francisco José; Font-Rotchés, Dolors (2007): «Entonación del español peninsular en habla espontánea: patrones melódicos y márgenes de dispersión». Moenia 13, 69-92.

Cantero, Francisco José; Mateo, Miguel (2011): «Análisis melódico del habla: complejidad y entonación en el discurso». Oralia 14, 105-128.

Cutler, Anne (1974): «On saying what you mean without meaning what you say». En LaGaly, Michael W.; Fox, Robert A.; Bruck, Anthony (eds.), Papers from the Tenth Regional Meeting. Chicago: Chicago Linguistic Society, 117-127.

Fónagy, Ivan; Magdics, Klara (1963): «Emotional patterns in intonation and music». Zeitschrift für Phonetik 16, 293-326.

Hidalgo, Antonio (2009): «Modalización (des)cortés y prosodia: estado de la cuestión en el ámbito hispánico». Boletín de Filología de la Universidad de Chile 44 (1), 161-195.

Hidalgo, Antonio (2011). «Humor, prosodia e intensificación pragmática en la conversación coloquial española». Verba 38, 271-292.

Hidalgo, Antonio (2016): «El estudio de la entonación del español hablado: una visión retrospectiva en el umbral del S. XXI». En Fernández Planas, Ana María (ed.), 53 reflexiones sobre aspectos de la fonética y otros temas de lingüística. Barcelona: Laboratori de Fonètica de la Universitat de Barcelona, 223-231 (http://stel.ub.edu/labfon/amper/homenaje-eugenio-martinezceldran/53reflexiones.html)

Hidalgo, Antonio (2017): «Nuevas aportaciones al estudio funcional de la entonación coloquial: propuesta ecléctica de integración de modelos de análisis». Estudios Filológicos 60, $127-150$.

Hidalgo, Antonio (2019): Sistema y uso de la entonación en español hablado. Aproximación interactivo-funcional. Santiago de Chile: Universidad Alberto Hurtado Ediciones.

Kienpointer, Manfred (1997): «Varieties of rudeness: types and functions of impolite utterance». Functions of Language 4 (2), 251-287.

Léon, Pierre (1970): «Systématique des fonctions expressives de l'intonation». Studia Phonetica 3, 57-74.

Léon, Pierre (1971): Essais de phonostylistique. Montréal: Marcel Didier.

Léon, Pierre (1972): «Patrons expressifs de l'intonation». Acta Universitatis Carolinae. Philologica I. Phonetica Pragensia 3, 149-155.

Léon, Pierre; Martin, Philippe (1970): Prolégomènes à l'étude des structures entonatives. Montréal: Marcel Didier.

Martínez, Hernán. y Rojas, Darcy (2011): «Prosodia y emociones: datos acústicos, velocidad de habla y percepción de un corpus actuado». Lengua y habla 15, 59-72.

Muecke, Douglas C. (1978): «Irony Markers». Poetics 7, 363-375. 
Myers Roy, Alice (1977): «Towards a definition of irony». En Fasold, Ralph W.; Shuy, Roger W. (eds.), Studies in Language Variation. Washington: Georgetown University Press, 171-183.

Padilla, Xose (2004): «El tono irónico: estudio fonopragmático». Español Actual 81, 85-98.

Padilla, Xose (2009): «Marcas acústico-melódicas: el tono irónico». En Ruiz, Leonor; Padilla, Xose (eds.), Dime cómo ironizas y te diré quién eres. Frankfurt: Peter Lang, 135-166.

Padilla, Xose (2011): «¿Existen rasgos prosódicos objetivos en los enunciados irónicos?». Oralia 14, 203-227.

Plutchik, Robert (1980): Emotion: Theory, research, and experience: Vol. 1. Theories of emotion. New York: Academic Press.

Schaffer, Rachel (1981): «Are there consistent vocal clues for irony?» En Masek, Carrie S.; Hendrick, Roberta A.; Miller, Mary Frances (eds.), Papers from the Parasession on Language and Behavior. Chicago, IL: Chicago Linguistic Society, 204-210.

Truesdale, Deirdre M.; Pell, Marc D. (2018): «The Sound of Passion and Indiference». Speech Communication 99, 124-134. 